\title{
Mechanical behavior of beams with variable stiffness obtained by 3D printing
}

\author{
Laszlo Racz ${ }^{1}$, and Mircea Cristian Dudescu, ${ }^{1, *}$ \\ ${ }^{1}$ Technical University of Cluj-Napoca, Department of Mechanical Engineering, B-dul Muncii 103- \\ 105, Cluj-Napoca, Romania
}

\begin{abstract}
Additive manufacturing or $3 \mathrm{D}$ printing gained a widespread popularity in recent years due to the ability of the method to manufacture components with high geometrical complexity. The most cost-effective process to manufacture plastic parts using 3D printing is the fused deposition modeling (FDM) method. Process parameters as the infill rates but also the printed pattern of different layers and their orientation have a significant influence on the mechanical properties of specimens fabricated by FDM. Controlling the process parameters is possible to generate materials with variable mechanical proprieties. The paper presents the analysis of a beam with constant cross-section but variable stiffness. Variable stiffness is achieved by changes in different cross-sections of the beam of the infill rates of the printing process. The mechanical behavior consisting of force-displacements curves is analyzed by three-point bending tests of variable stiffness samples and comparison with similar beams having constant infill rate. The results consist of E-modulus variation, maximum force and deflection curve. Analytical calculations and finite element analyses are employed to predict the mechanical behavior of the specimens printed with variable infill rate. The obtained results proved the concept of equal stress-beam with constant cross-section obtained by $3 \mathrm{D}$ printing process parameters variation.
\end{abstract}

\section{Introduction}

Additive manufacturing and particularly $3 \mathrm{D}$ printing gained a widespread popularity in recent years due to the ability of the method to manufacture components with high geometrical complexity. The most cost-effective process to manufacture plastic parts using 3D printing is the fused deposition modeling (FDM) method. Beside the complex geometries that can be achieved one important aspect are the mechanical proprieties of the printed parts. The mechanical properties of specimens fabricated by fused deposition modelling are significantly influenced by

* Corresponding author: mircea.dudescu@rezi.utcluj.ro 
the printing parameters. Several studies [1-4] demonstrated by experimental or numerical methods the influence of the infill rates but also the printed pattern of different layers and their orientation upon the mechanical behavior of printed parts. A valuable approach for mechanical property optimization of fused deposition modeling with polylactic acid via design of experiments is described in [5]. The methodology implied a two-factor-level Taguchi test matrix defined to ensure mechanical testing of several different fabrication settings using a reduced array of experiments. Investigation into the effect of air gap, raster width, raster angle, contour number and contour width, with a full factorial design of the experiment on the flexural properties of FDM-produced parts are presented in [6]. The obtained results revealed that raster angle and raster width have the greatest effect on the flexural properties of the material, the paper presenting also the optimal levels of the process parameters. Other research [7] presents an experimental investigation and optimization of FDM process variables using a hybrid statistical approach to attain desired flexural strength. The hybrid approach combines response surface methodology and genetic algorithm to predict flexural strength of FDM fabricated samples.

Creating models with variable infill rate and infill pattern would present a series of benefits compared to conventional 3D printed models. First of all, a better weight - strength ratio could be obtained by optimizing the internal structure to fit the requirements of utilization. In the less stress areas, the infill rate can be lowered, and in the areas of high stress the infill rate can be increased to better withstand the loads. By doing so, significant amount of material and printing time can be spared. The mechanical behavior of the 3D printed parts is also influenced by infill pattern in combination with the infill rate. To exploit the full potential of creating customized internal structures in $3 \mathrm{D}$ printed parts according to load distribution, a suitable modeling method should be established. With this procedure it would be theoretically possible to create parts with optimum dimensions in terms of geometrical shape and optimized internal structure.

Unfortunately, there is no suitable 3D printing tool at the moment which facilitates variable infill rate and infill pattern creation. The most advanced, from this point of view is Simplify 3D software, which allows to work with multiple parts and multiples settings in one printing session. It has also a possibility to configure settings in a manner, to obtain a 3D printed part with variable infill rate. The feature which makes this possible is to create different printing configurations for different heights, by defining the position where the printer will start or stop to print, according to a specific configuration. For our study we proposed to create a test specimen, to study the influence of the variable infill on the mechanical behavior of the 3D printed part.

\section{Materials and methods}

\subsection{Sample manufacturing}

As it was mentioned before, there is no standard feature in $3 \mathrm{D}$ printing software to determine the areas of the different infill rate or infill pattern. In order to create models with variable infill rate or infill pattern, we had to come up with a method which permits to set different printing configuration within one part. The tested solution was, to divide a geometrical volume into subvolumes. The sub-volumes are necessary to clearly define the delimitations within one part, where the different printer configuration can be applied. In Simplify 3D software (Simplify3D Software, Cincinnati, USA), there is a possibility to define individual printing parameters to a previously created sub-volume defines as a "new model". For our study we proposed to analyze the effect of infill rate on 3D printed part printed with $0^{\circ}$ direction (according to the longitudinal axis of the sample) and variable infill rate. 
The variation of the infill rate will be $20 \%-40 \%-60 \%-80 \%-100 \%-80 \%-60 \%-40 \%-20 \%$. The reason why this distribution of infill rate is chosen: the part will be loaded at the middle in a testing machine (Instron 3366, USA) and the load and flexure extension will be measured by doing a 3-point bending test (Fig. 1).

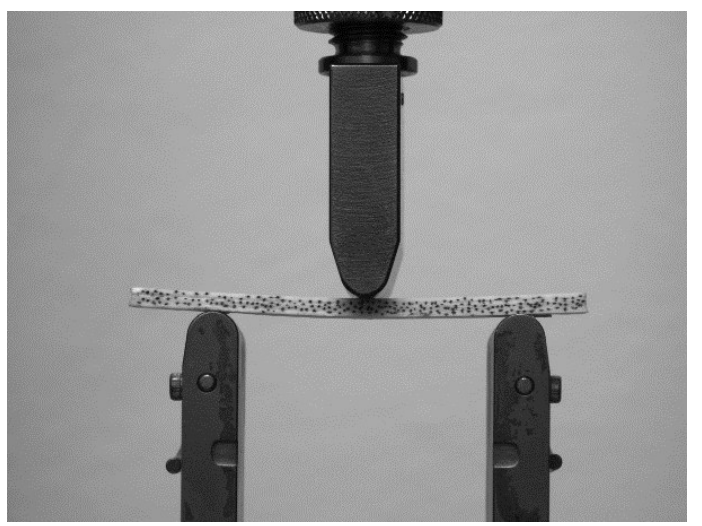

Fig.1. Three point bending test setup for 3D printed specimen with variable infill rate.

The results of the measurements then will be compared with the result of another set of printed specimens with a uniform $100 \%$ infill rate 0 o printing direction -according to the longitudinal axis of the test specimen. First the geometrical model of the specimen was created in SolidWorks and exported to the preprocessing tool of the printer. The created test specimen, (with the following dimensions: $90 \mathrm{~mm}$ long, $4 \mathrm{~mm}$ height, $10 \mathrm{~mm}$ width) had to be divided, in nine sections as presented din Fig. 2.

The sections were defined as "new models", so the software treats them individually, thus allowing to set different printing configurations for each "new model". As the "new models" are defined, the software allows the user to define different infill rate or different infill pattern for each model as seen in Fig. 2 a).

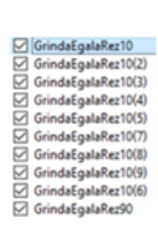

a)

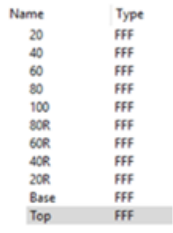

b)

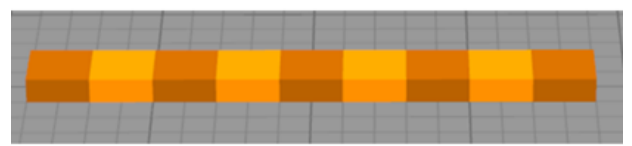

c)

Fig. 2. Settings of the test specimen a) sub-model settings, b) process settings, c) geometrical definition of the sub-models.

By double clicking on the model definition list, the full range of settings are available for the user to choose from. The disadvantage of this modeling approach is that the software will try performing a regular printing process for each individually defined submodel, where in standard mode the shell section of the part is built also. This feature is facilitating different printing parameter application in parts oriented vertically on the 
printing platform, where the different parameters are possible to be changed according to the height of the printing nozzle.

The solution used in this study was to define two more processes and to add one more geometry, which was used to create the base - top and the shell section of the model as presented in the Fig. 2 b). The added extra geometry was used to print the bottom and the top section of the test sample- where continuous layers were needed- then the printer started to print the individual sections with the variable infill rate, and finished it again with a continuous printing of the top layers, using the same geometry as for the basis. The process list and the model list are synchronized, and the starting and ending point of different processes could be set up this way. Before starting the printing, the user has the option to continue with "layer-by-layer" printing or "object-by-object". The layer-by-layer printing means that the printer will build up the parts layer-by-layer regardless of the setting and number of parts. The printing will start with the process defined as "Base" and will continue to go through all the models and assigned processes without building the individual models with a specific process individually. The object-by-object settings will command the printer to print each "new model" individually and only upon completion will go to perform the same operation for the next "new model" or as it is named in this case: object.

One issue is still not eliminated completely: the lines are interrupted by internal shells which does not permit to have continuous lines across the entire length of the test specimen as presented in the Fig. 3. The partition areas, which are practically shell area of the individual models are marked with red on the picture. The fact that we don't have an entirely continuous line probably will reduce the deflection of the test specimen. Complete deactivation of the shell creation would lead to increase of air gaps in the part which would significantly influence the mechanical behavior of the sample, so it was not an option. Although this problem is foreseen, the investigation was continued in order to see the effect of this configuration on the behavior of the test specimen.

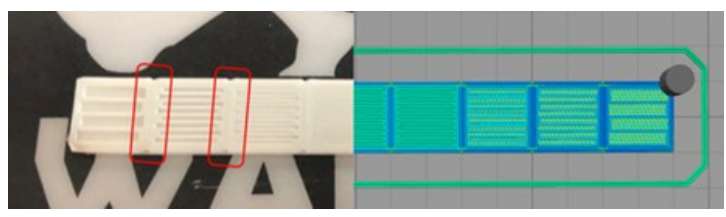

Fig. 3. Test specimen during the printing process with highlighted internal thin walls between different settings. (Left side: printed specimen, right side: printer settings).

The next effort was made in the direction to completely eliminate the partition areas between different printer setting in order to create a model with continuous lines across the length of the whole part. To create such a model, the length of the geometrical sub models was modified, extended with a half of the "internal thin wall" partition area. Doing so the printing software was able to print the crossing from one area with a different setting to another without building an internal thin wall as presented in the Fig. 4.

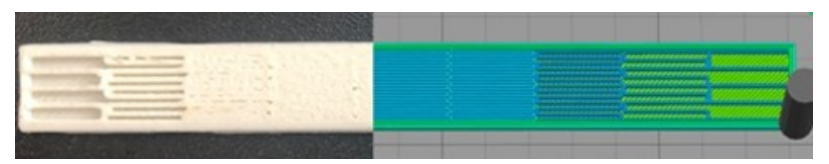

Fig.4. Improved test specimen during the printing process without generating thin walls between different settings. (Left side: printed specimen, right side: printer settings). 


\subsection{Experimental study}

Three sets of test specimens were printed (with FDM method using ABS material) and tested. One set was printed with regular uniform settings, using $100 \%$ infill ratio and grid pattern, $0^{\circ}$ direction according to the longitudinal axes of the test samples. The second set of test specimens were printed with variable infill ratio, (20\%-40\%-60\%-80\%-100\%-80\%$60 \%-40 \%-20 \%$ ) as described previously, where between the different infill rate scenarios the dividing thin wall was kept. The third set of test samples were printed according to the same infill percentage distribution, but without the thin walls dividing the different infill scenarios. Each set of test specimen contained five 3D printed samples. The samples were tested with an Instron testing instrument for 3-point bending under a static load. The test results are presented in the Table 1.

Table 1. Three point bending test results of the printed specimens.

\begin{tabular}{|c|c|c|c|c|}
\hline \multirow{2}{*}{ Specimen types } & $\begin{array}{c}\text { Modulus } \\
\text { (Automatic) }\end{array}$ & $\begin{array}{c}\text { Flexure stress } \\
\text { at max. load }\end{array}$ & Flexure load & $\begin{array}{c}\text { Deflection at } \\
\text { max. load }\end{array}$ \\
\cline { 2 - 5 } & $(\mathrm{MPa})$ & $(\mathrm{MPa})$ & $(\mathrm{N})$ & $(\mathrm{mm})$ \\
\hline $\mathrm{U}$ & $1,984.00$ & 53.67 & 95.42 & 7.414 \\
\hline $\mathrm{V} 1$ & $2,078.20$ & 45.26 & 80.46 & 3.942 \\
\hline $\mathrm{V} 2$ & $2,004.40$ & 48.76 & 86.69 & 4.917 \\
\hline
\end{tabular}

A graphic representation of the deflection is presented in Fig. 5. where: U- represents the uniformly printed test specimen results, V1- presents the result of the test specimen with thin walls and variable infill rate, V2- represents the result of the specimens printed with variable infill, without thin walls.

Comparing the results of the tests we can conclude that the effect of the variable infill rate has a significant effect on the stiffness of the test specimen. There is a slightly lower deflection and slightly steeper curve presented in Fig. 5. for samples with the variable infill (V1) up to $\sim 65 \mathrm{~N}$, but suddenly they fail at $\sim 80 \mathrm{~N}$ load (min. $77.33 \mathrm{~N}$; $\max 83.1 \mathrm{~N}$ - range for the 5 specimens). The second set of test samples (V2), printed with variable infill rate, but without the thin walls between the different settings, performed better than the version with the thin wall V1, their load bearing capacity has increased with $7.75 \%$, failing eventually at $86.69 \mathrm{~N}$ ( $\min .84 .63 \mathrm{~N}$; max $92.32 \mathrm{~N}$ - range for the 5 specimens).

The test specimen printed with uniform settings (U) performed the best, withstanding load up to $\sim 95 \mathrm{~N}$ (min. 94.12; max. $96.55 \mathrm{~N}$ - range for the 5 specimens), which is $18 \%$ difference compared to specimen V1, and 9.5\% compared to specimen V2. The measured flexural modulus of the specimen with variable infill with the thin wall (V1) is the highest $2078(\mathrm{MPa})$ is slightly higher than in case of specimens printed with variable infill and without the thin wall (V2) with a mean value of $2004 \mathrm{MPa}$, compared to $1984 \mathrm{MPa}$ for the first specimen printed with uniform infill rate. (representing a difference of $4.75 \%$. between specimen $\mathrm{U}$ and specimen $\mathrm{V} 1$, and $1 \%$ between specimen $\mathrm{U}$, and specimen V2). The flexure extension difference between the two variable printed samples is very high. V2 extends $25 \%$ longer than V1. 


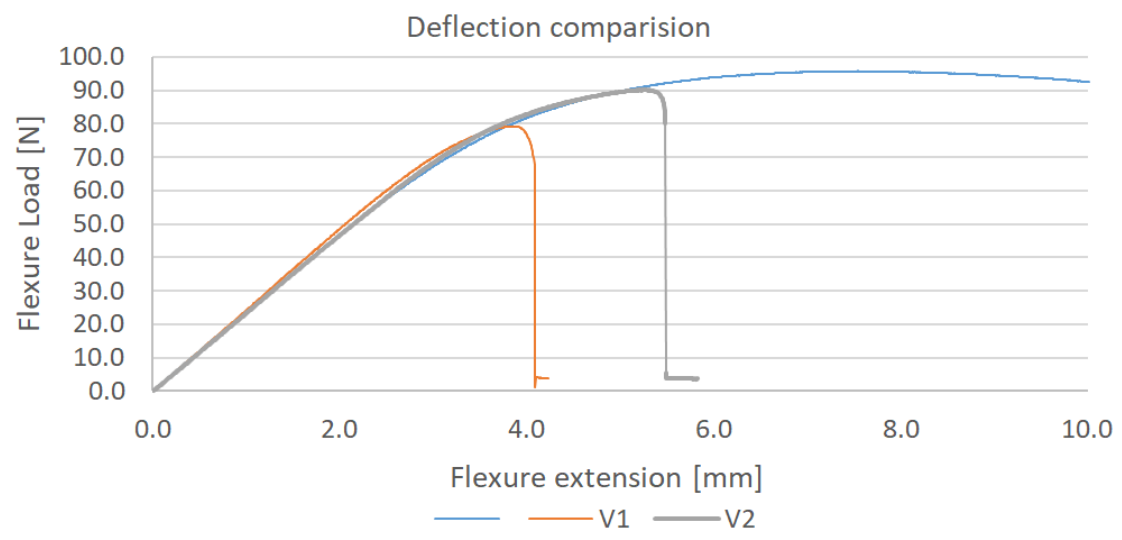

Fig. 5. Comparison of the uniformly and variably printed test specimen's flexure curves.

The specimen $U$ extends $51 \%$ more than the one printed with variable infill rate without the thin wall V2. This is probably due to the fact that the printed filament layers are not going continuously, they are interrupted by the partition zones between the variable infill sections. What is also an important factor: between the samples of the same set of test specimens the inconsistency in individual results is always higher in the case of the specimens printed with variable infill rate. In order to compare with the analytical model, respectively FE, force-deflection values are chosen from the elastic domain, a force of $60 \mathrm{~N}$ for which corresponds a deformation range (for 5 specimens) from 2.59-2.64 $\mathrm{mm}$. for $\mathrm{U}$, 2.45-2.52mm for V1 and 2.51-2.64- for V2.

\subsection{Finite Element analysis}

Finite Element (FE) analysis was conducted on the printed specimens to determine the accuracy in predicting the mechanical behavior of 3D printed parts using standard $\mathrm{FE}$ analysis. It is very difficult to predict the mechanical behavior of the $3 \mathrm{D}$ printed parts without testing them using experimental methods. Testing requires a lot of printing time and material usage. Predicting the mechanical properties of a functional FDM part before actually printing it could bring a series of advantages: material waste could be minimized, printing time could be optimized, influence of the printing parameters on mechanical properties of the part could be assessed and optimized before printing the part physically. In order to achieve a reliability in predicting the mechanical behavior of the printed specimens, FE analysis was carried out and results compared to the experimental findings. First the FE model of the test specimens were created in ANSA (BETA CAE Systems SA, Thessaloniki, Greece.) Two modelling approaches were used to determine the most suitable method for analyzing the 3D printed samples. The first modelling approach used Tetraida $2^{\text {nd }}$ order solid mesh, with dimensions of the elements ranging from $0.5-1.5 \mathrm{~mm}$. The model was divided in nine sections, different material setting was applied for the variable infill rate. Using the result form, a previous experimental study where the Tensile Modulus for different infill rates was experimentally determined, and numerically adjusted to the correct cross section- the FE models variable infill rate was represented as a difference in material with a modulus corresponding to a specific infill rate. The model was loaded with a $60 \mathrm{~N}$ force, applied in the middle of the test specimen. Element quality was checked for standard NASTRAN requirements. Standard linear-static simulation was performed on the model using Epylisis (BETA CAE Systems SA, Thessaloniki, Greece.) solver. The postprocessing of the data was done in Metapost (BETA CAE Systems SA, Thessaloniki, 
Greece.). The results are shown in Fig. 6. where a) represents the deflection according to direction of the load $2.787 \mathrm{~mm}, \mathrm{~b}$ ) visual representation of the stress distribution

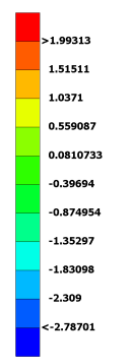

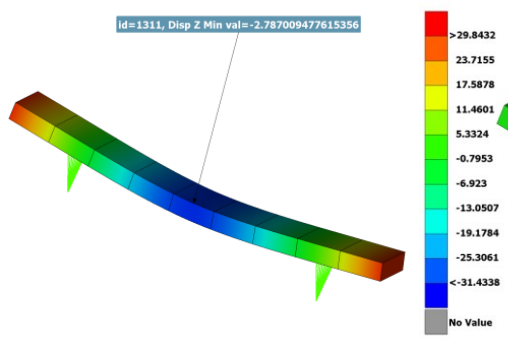

a)

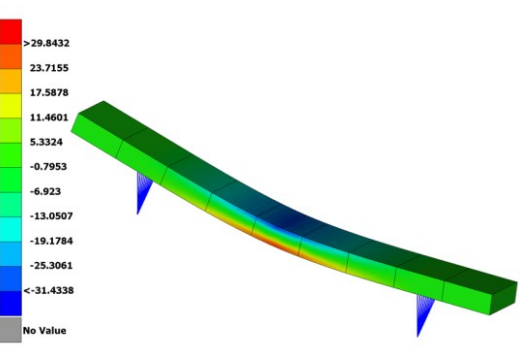

b)

Fig. 6. Results of the FEM analysis of the Tetraida model; a) visual representation of the flexural extension, b) visual representation of the normal stress distribution .

The second modelling approach was to build up the test samples using Hexaida elements. Using the same element quality requirements and dimensions as for the Tetraida model, same load and material settings the model was simulated and compared to the Tetraida model. In the Fig. 7 the result of the FEM simulation of the Hexaida model is presented where a) represents the deflection according to direction of the load $2.770 \mathrm{~mm}, \mathrm{~b}$ ) visual representation of the stress distribution

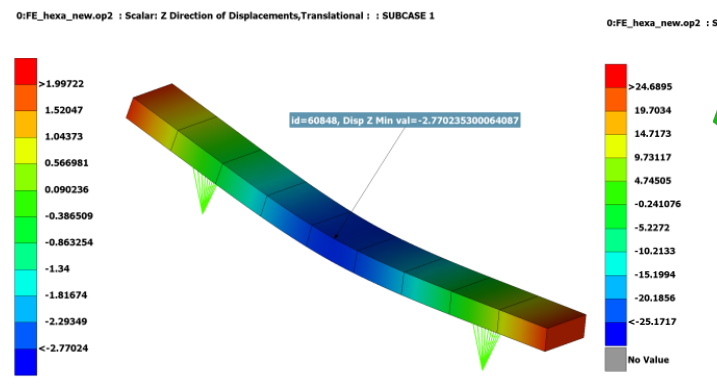

a)

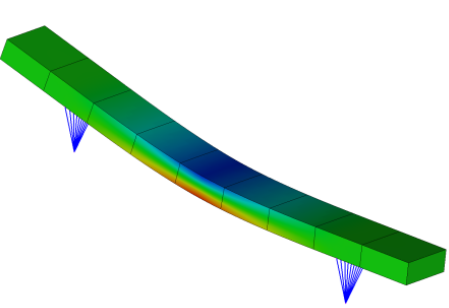

b)

Fig. 7. Results of the FEM analysis of the Hexaida model; a) visual representation of the flexural extension, b) visual representation of the normal stress distribution.

It can be noticed that the results are almost the same, having only $\sim 0.4 \%$ deviation between the result. Comparing the results of the two models gives us insight about the precision and running time. The running time for the more complex Tetraida second order model was 10.8 seconds, while the Hexaida model was compiled in 1.5 seconds. For simple geometrical shapes the Hexaida model delivered faster and more accurate result then Tetraida. Both methods delivered acceptable result, although in case of analyzing a part with a complex geometrical shape, a bigger divergence in result can be expected due to the fact that the solver can approximate the results better using a Tetraida second model, utilizing a larger node number.

\subsection{Analytical calculation}

Calculation of the analytical beam's deflection has been done by energy methods, the Castigliano's second theorem. The calculation scheme (free body diagram) is presented in 
Fig. 8 and consist of a simply supported beam with a concentrated force placed at the half distance of the beam span. Different regions of the beam having E-moduli $\mathrm{E}_{2}-\mathrm{E}_{5}$ are marked by their span. The dimension as for experimental analysis are chosen: $a=5 \mathrm{~mm}$, the halflength $l=30 \mathrm{~mm}, F=60 \mathrm{~N}$. The E-moduli correspond to different infill rates, were determined experimentally, where: $\mathrm{E}_{1}=1901 \mathrm{MPa}$ - infill rate $20 \% ; \mathrm{E}_{2}=1974 \mathrm{MPa}$ - infill rate $40 \% ; \mathrm{E}_{3}=2052 \mathrm{MPa}$ - infill rate $60 \% ; \mathrm{E}_{4}=1983 \mathrm{MPa}$ - infill rate $80 \% ; \mathrm{E}_{5}=1703 \mathrm{MPa}$ - infill rate $100 \%$. The portion with E1 is outside of support, which is why it does not appear in the analytical model. The cross-sectional dimensions are width of $10 \mathrm{~mm}$ and thickness of $4 \mathrm{~mm}$.

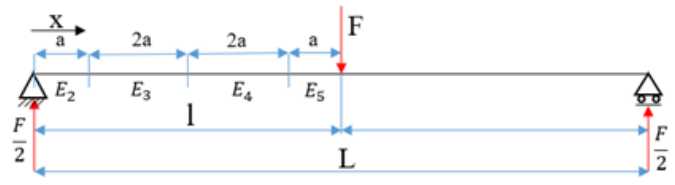

Fig. 8. Schematic representation of the analytic model.

According to Castigliano's second theorem the deflection in the load application point can be expressed considering the derivative of the bending moment with respect to load:

$$
\delta=\int \frac{M}{E_{I}} \cdot \frac{\partial M}{\partial F} \cdot d x
$$

In our case, considering four different intervals with different E-moduli and the same moment of inertia (I) we get:

$$
\begin{gathered}
\delta=2\left(\int_{0}^{a} \frac{F}{\frac{2}{E_{2} \cdot I}} \cdot \frac{x}{2} \cdot d x+\int_{a}^{3 a} \frac{\frac{F}{2} \cdot x}{E_{3} \cdot I} \cdot \frac{x}{2} \cdot d x+\int_{3 a}^{5 a} \frac{\frac{F}{2} \cdot x}{E_{4} \cdot I} \cdot \frac{x}{2} \cdot d x+\int_{5 a}^{6 a} \frac{\frac{F}{2} \cdot x}{E_{5} \cdot I} \cdot \frac{x}{2} \cdot d x\right) \\
\delta=\frac{2 F}{4 I}\left(\frac{x^{3}}{E_{2} 3} \int_{0}^{a}+\frac{x^{3}}{E_{3} 3} /_{a}^{3 a}+\frac{x^{3}}{E_{4} 3} /_{3 a}^{5 a}+\frac{x^{3}}{E_{5} 3} /_{5 a}^{6 a}\right) \\
\delta=\frac{F}{2 I}\left(\frac{a^{3}}{3 E_{2}}+\frac{8 a^{3}}{3 E_{3}}+\frac{116 a^{3}}{3 E_{4}}+\frac{91 a^{3}}{3 E_{5}}\right)
\end{gathered}
$$

In case of a constant E-modulus the above formula becomes the known formula for the deflection of the midpoint of a simply supported beam loaded by a concentrated force.

$$
\delta=\frac{F l^{3}}{6 E I}
$$

Replacing the above dimensions and material constants we get a deflection value of $2.72 \mathrm{~mm}$.

\section{Conclusions}

The effect of the variable infill rate on the mechanical behavior of the 3D printed ABS parts was studied. In order to create $3 \mathrm{D}$ printed parts with variable infill, a model creation method had to be established. For establishing the printing parameters Simplify 3D software was employed, which allows the user to assign different printing parameters to previously defined volumes. Three sets of five test specimens were printed and tested under a three-point bending test. One set of test specimens was printed with a uniform infill rate of $100 \%$, direction of printing 0 degree, according to the longitudinal axis of the specimen. The second and third set of test specimen were printed with a variable infill rate $(20 \%-40 \%$ - 
$60 \%-80 \%-100 \%-80 \%-60 \%-40 \%-20 \%$ ). The difference between the two variable printed specimens (referred to as V1) consists in an improved transition zone between the areas with the infill rate changes (referred to as V2). Comparing the results of the tests we can conclude that the variable infill rate has a significant effect on mechanical behavior of the specimens. The revised modeling method improved the load bearing capabilities of the variable printed specimens with $7.5 \%$ from $80.46 \mathrm{~N}$ to $86 \mathrm{~N}$. Also, the flexure extension increased $25 \%$ from $3.9 \mathrm{~mm}$ to $4.9 \mathrm{~mm}$. The specimen printed with uniform $100 \%$ infill rate $\mathrm{U}$ performed the best, both from strength and extension point of view. Considering the usage of the material between the uniformly printed samples $U$ and variably printed $V 2$, the material saving is $\sim 13 \%$ (1210 mm instead of $1350 \mathrm{~mm}$ filament length). The difference in maximum flexure load is $\sim 10 \%(86 \mathrm{~N}$ - to $95 \mathrm{~N})$, while the flexure extension difference is a significant $51 \%$ (4.9mm to $7.4 \mathrm{~mm})$. The models were also analyzed with the finite element method. Two modelling approaches were used to determine the most suitable method to accurately predict the mechanical behavior of the test specimens using FE analysis. The difference between the two models where insignificant, but the Hexaida models result was closer to the experimental result, predicting a displacement of $2.77 \mathrm{~mm}$. (2,787 Tetraida model) The experimentally determined flexure extension was between $2.51-2.64 \mathrm{~mm}$ for the five tested V2 specimens. The results show a deviation of $4.9-10.3 \%$ between the experimental values and the results obtained with FE analysis. The displacement of the improved, variable printed specimen V2 was calculated also analytically. The calculated displacement was $2.72 \mathrm{~mm}$, which is $3-8.3 \%$ deviation from range of the experimental result. Also, an increase in inconsistency of the experimental result can be noticed in case of the variable printed test specimens, leading to larger unpredictability in results, compared to the uniformly printed specimen. Variable printed FDM parts could become a cheap and effective way to create highly optimized parts in term of weight -strength ratio, keeping material usage at minimum

\section{References}

1. M. L. Dezaki, M. K. A. M. Ariffin, and S. Hatami, Rapid Prototyp. J., (2021)

2. A. Qattawi, J. Manuf. Process., 36, 164-174, (2018)

3. M. Lalegani Dezaki and M. K. A. Mohd Ariffin, Polymers (Basel)., 12, 12, 2792, (2020)

4. C. Dudescu, L. Racz, ACTA Univ. Cibiniensis, 69, 1, 23-30, (2017)

5. J. Torres, M. Cole, A. Owji, Z. DeMastry, and A. P. Gordon, Rapid Prototyp. J., (2016)

6. A. W. Gebisa and H. G. Lemu, Materials (Basel)., 11, 4, 500, (2018)

7. S. Dev and R. Srivastava, Mater. Today Proc., (2021) 